\title{
A Summit of Kick-Ass Physics Students
}

\author{
Hundreds of budding physicists from 42 countries gathered in a virtual \\ edition of PLANCKS - a student competition that hands out prizes \\ proportional to the Planck constant.
}

\author{
By Matteo Rini
}

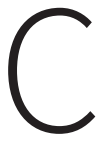
an you derive a model for the expansion of the Universe from Newton's gravitational laws? Can you also estimate the maximum data-transmission rate through a 300-km-long optical fiber and devise a way to use a soap bubble as a barometer? And could you solve these and tens of other brain-racking problems all within a day and a half, while attending a busy international meeting and mingling with hundreds of your peers?

These were the brutal demands placed on bachelor's and master's degree physics students participating in the 2021 Physics League Across Numerous Countries for Kick-Ass Students (PLANCKS). The taxing efforts were matched by

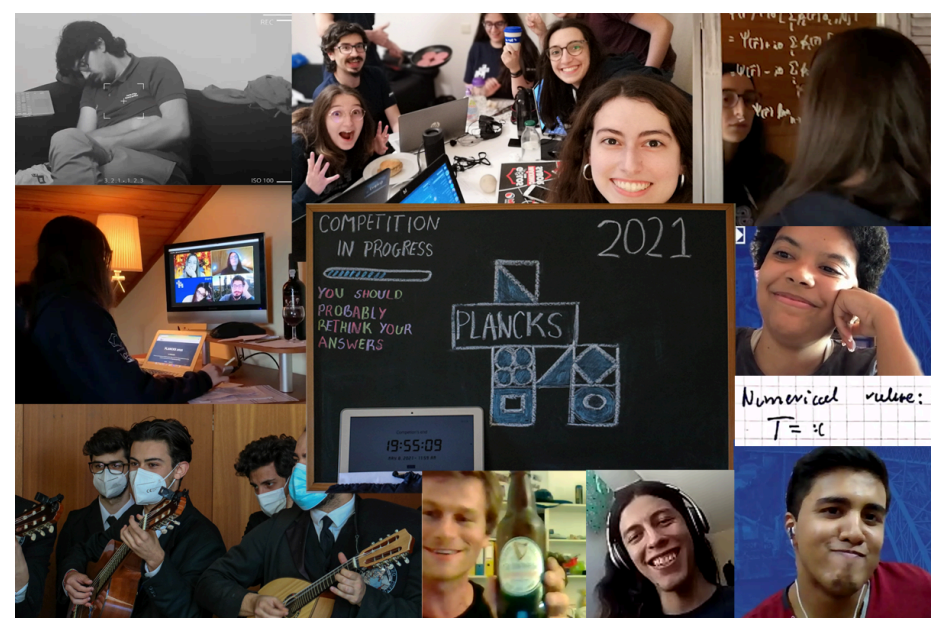

Scenes from the 2021 PLANCKS competition for physics students from around the globe.

Credit: Márcio Lima; PLANCKS2021 quantum-sized rewards, with winning teams earning monetary prizes that were multiples of the Planck constant.

The International Association of Physics Students launched PLANCKS in 2014 with the goal of allowing students from all over the world to get in touch with each other, to enjoy opportunities for personal development, and to experience, early on, the spirit of international collaboration that is at the core of a physicist's life. Participants work in teams to solve theoretical problems from all fields of physics, but they also attend a number of activities, from seminars by scientific celebrities to social events and excursions that showcase the research environment and the culture of the host country.

Each year, an institution in a different country organizes the event. The University of Porto in Portugal was this year's host, but the ongoing pandemic forced the competition to be online. The virtual setting was a "curse and a blessing," says Duarte Graça, one of the local organizers. He says that the online format allowed the presence of many students who couldn't have afforded an international trip. This year's 200 participants came from 6 continents and 42 countries-twice as many countries than in previous years. Some of the competitors received "global grants" covering the registration fee. "When we watched their grant-application videos, we realized how much [being there] mattered to them," says Sofia Ferreira Teixeira, another member of the organizing team.

For most virtual conferences in the time of COVID-19, increased accessibility comes at a cost-lack of impromptu interactions, Zoom fatigue, and a dearth of networking opportunities. It was 
hard to note any of those shortcomings at PLANCKS, with energetic students and sleep-deprived organizers continually popping in and out of breakout rooms that were open around the clock to accommodate the time zones of all participants. In "warm up" sessions at the start of the event, the locals trained everyone on Portuguese culture and student life, showcasing architecture and natural riches, explaining graduation ceremonies and freshman-hazing rituals, or simply teaching the difference between a "fino" and a "caneca" (a small and a large beer).

During "Nations Coffee Breaks," students proudly presented treasures from their respective countries, with some of the highlights being Venezuela's national dance, joropo; recipes for a Mexican sancocho soup; pristine beaches in Mozambique; lavish saunas in Finland; and the Brazilian sounds of samba, bossa nova, and funk. But the participants also found common ground in aspects of their physics-student lives, from the pleasure of eating unhealthy "lab snacks" to the difficulty of explaining one's own research to Grandma.

The event always features talks by top scientists, who can easily be convinced to engage with a passionate, young audience-Stephen Hawking attended the 2014 inaugural edition. This year, students heard about space plasma, gravitational-wave astronomy, nuclear fusion, quantum information, and transparent electronics. Physics magazine offered a workshop on science writing and communication.

But a student favorite was astrophysicist Jocelyn Bell Burnell, who discovered the first radio pulsar in 1967. Bell Burnell spoke about her scientific work but also opened up about the psychological challenges she faced when she moved from Scotland to Cambridge-or, from "a land of uncouth savages" to the "area of ultimate civilization," according to perceptions in the South of England at that time. "As scientists we don't often talk about our stories, but life stories can be really helpful to those who may feel a bit daunted," says Bell Burnell. She is particularly keen on offering encouragement and support to those who are in a minority-women and people of underrepresented ethnicities.

The final ceremony crowned three teams, who earned $\hbar / 2$, $\hbar / 3$, and $\hbar / 4$ times $10^{37}$ euros, respectively. A team of students from the University of Oxford grabbed the first prize ( $\mathrm{€} € 527$ bounty), but all students took home valuable memories. "It's really cool to hear about the breadth of different problems in physics," says Jose Betancourt from the University of the Andes in Colombia. "The joy of teamwork is what I will remember," says Siao Xiang of Nanyang Technological University, Singapore. "Making new friends and getting to know their countries and cultures was amazing," says Rasha Abukeshek from An-Najah National University in Palestine.

If you'd like to test your physics stamina, problems from past PLANCKS editions can be found here. But if you are a career physicist-or a Physics magazine editor-we do recommend that you keep your scores to yourself.

Matteo Rini is the Editor of Physics. 